\title{
Synthesis of Vibration Damping Control Systems based on the Identified Model of a Mechanical Construction at Broadband Disturbance Influence
}

\author{
Marygin Oleksiy \\ College of Aerospace Engineering, \\ State Key Laboratory of Mechanics and \\ Control of Mechanical Structures, \\ Nanjing University of \\ Aeronautics and Astronautics, \\ No. 29 Yudao Street, \\ Nanjing 210016, China.
}

\author{
Xing Shen \\ College of Aerospace Engineering, \\ State Key Laboratory of Mechanics and \\ Control of Mechanical Structures, \\ Nanjing University of \\ Aeronautics and Astronautics, \\ No. 29 Yudao Street, \\ Nanjing 210016, China.
}

\begin{abstract}
The changing of certain types of energy into other types in machines and mechanisms, the transformation of movement forms and carrying out certain working processes inevitably causes the emergence of variable forces and vibration-causing momentum. Vibration control and management are important tasks in aerospace field. This thesis solves the problem of the development of an effective control system based on the active method used for vibration reduction in a mechanical construction of a cantilever fixed metal plate broadband disturbance lab device. In the system synthesis process a carry out structural and parametric identification of the mathematical model of the controlled object using experimental data. LabVIEW 10.0 development environment and FPGA hardware technology from National Instruments were used to create a spectrum analyzer measurement system, which allows solving problems of experimental acquisition and identification of the dynamic properties of mechanical objects. With the help of MATHLAB environment mathematical apparatus and Optimization and Control System Toolbox packages, a mathematical model of the control object, regulators' synthesis was built; their optimal parameters were searched for, and system operation with different object structures and regulators (PID, LQG) was modeled.
\end{abstract}

\section{Keywords}

Control system, regulator, PID, LQG, vibration, control object, mathematical model, LabVIEW, MATHLAB.

\section{INTRODUCTION}

Modern aerospace aircraft are lightweight and are highly durable. Their speed and acceleration requirements are getting higher and higher, which causes vibration increase and the necessity of taking it into account when drafting aircraft blueprints. A desire to reduce the weight of the aircraft mechanical system doesn't allow for the usage of common damping materials only. Today one of the ways for vibration reduction is claimed to be the usage of piezoelectric materials [3,5]. Piezoelectric materials (PZT) possess physical and technological properties, which are important for the aircraft, that is: small volume, light weight, mechanical simplicity and the easiness of flexible structure integration. Because of direct and reverse piezoelectric effect, those materials are used as sensors and as executive mechanisms in structural vibration control systems. Today passive, semi-passive and active vibration damping methods are in development. [1,2].

\section{EXISTING PRINCIPLES OF VIBRATION CONTROL}

The main principle of the passive methods is that the energy from piezoelectric material deformation dissipates on shunt circuits - RLC chains. Energy dissipation process prevents piezoelectric material deformation and the structural damping increases $[2,3,4]$. Semi-passive methods of vibration damping use external power sources, the energy from which helps amplify the signal taken from piezomaterial and transmit it in the anti-phase to the same pzt patch $[2,3,4]$. Active methods use control systems based on feedback principle with a regulating device. Those systems are either built in analogue form with operational amplifiers and P, PI, PID control principles at their basis; or in discrete form with digital schemes and different control principles and regulation criteria $[2,3]$. This thesis presents building an active system of vibration control using digital controllers.

\section{ELECTROMECHANICAL SYSTEM AS A CONTROL OBJECT}

As a control object, this thesis uses a cantilever fixed metal plate the vibration of which needs to be reduced with the help of an executive mechanism and a pzt patch sensor. The aluminum plate has dimensions of $300 \times 40 \times 1 \mathrm{~mm}$; its elastic modulus is $70 \mathrm{GPa}$; its density is $2700 \mathrm{~kg} / \mathrm{m}^{3}$. The data above allows for calculation of its resonance frequencies $(9.1,57$, $160,314,523 \mathrm{~Hz}$ ) and use them as reference points when determining the dynamic properties of the object. Analytical determination of a complex "mechanical system - pzt converter" structure is quite a complicated task; besides, the presence of inhomogeneity in the mechanical structure, deviation from the form and the introduction of additional masses - pzt sensor and actuator - drastically change the dynamics of the object. Thusly an important direction in studying the behavior of a mechanical object is the experimental determination of its dynamic qualities [11,12]. The task set in this thesis is to reduce the vibration level of a mechanical system, where the vibration is induced by HEV50 electromechanical exciter using manipulating influence via a PZT actuator (Fig. 1). 


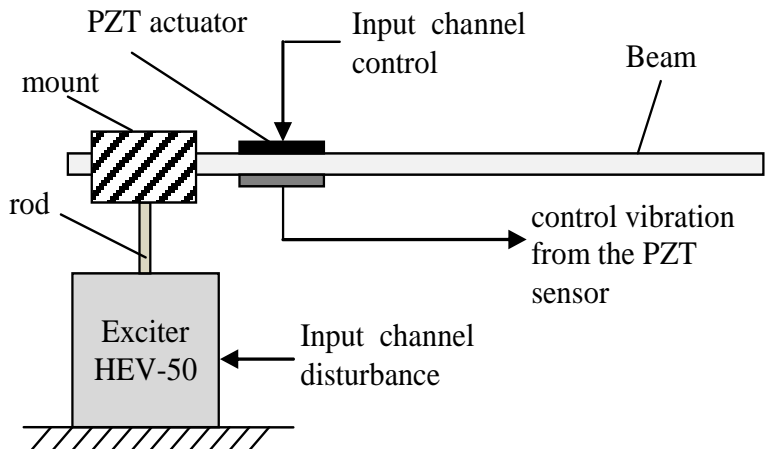

Fig. 1: Allotment of control and disturbance channels of the research object.

The analysis of the aforementioned electromechanical system as the control object allows to allot disturbance and control channels. In the disturbance channel, force $f$ generated by HEV-50 influences the mechanical system and leads to its disturbance $X_{l}$. In the control channel, the signal sent to the pzt actuator causes its flex and the flex of the plate $X_{2}$ proportionally to the level of voltage applied $U(t)$. The flex of the pzt actuator should level out the flex of the plate caused by the disturbance. Each of the allotted channels has its own dynamic characteristics, which need to be defined for the further control system synthesis (Fig. 2).

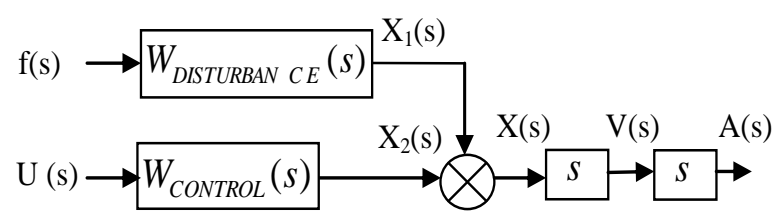

Fig. 2: General structural scheme of the object

\section{OBJECT DYNAMIC CHARACTERISTICS MEASUREMENT METHOD USED}

A dual-channel spectrum analyzer was used for the definition of the dynamic properties of the control object. This method is based on the spectral analysis of the stimulating signal and response assuming the linearity of the electromechanic system. The research signal used was of the "white noise" type. System response of the acceleration type was measured with a pzt sensor. The working principle of the analyzer is represented in fig. 3 .

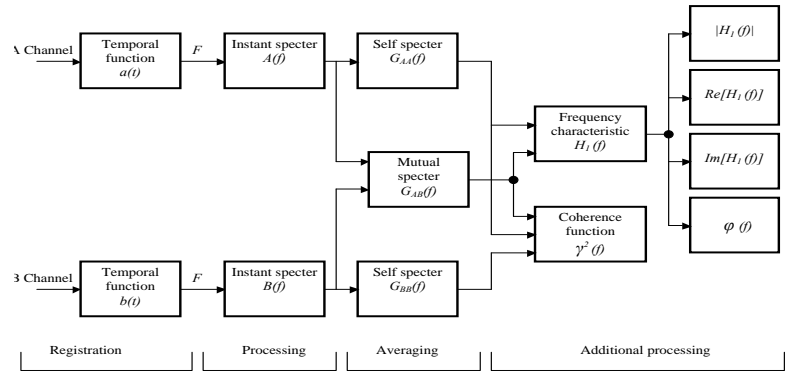

Fig. 3: Principal block-scheme of a program dual-channel spectrum analyzer.

Signals $a(t), b(t)$ applied to the analyzer's inputs are changed to the digital form $a[n k], b[n k]$ so as to get the series of digital data (realizations). Recorded realizations are transformed into frequency domain as complex instant specters $A(f)$ and $B(f)$ using discrete Fourier transform [6,7,8]. Mutual and self specters of the stimulating signal and reaction $G_{A A}(f)$, $G_{B B}(f)$, are the functions, necessary for the frequency characteristics assessment, and coherence functions.

Coherence function shows the degree of linear dependence for accounted signals in the frequency domain. Functions can be presented as

$$
H_{1}(f)=\frac{G_{A B}(f)}{G_{A A}(f)}, \quad H_{2}(f)=\frac{G_{B B}(f)}{G_{B A}(f)}, \quad \gamma^{2}(f)=\frac{\left|G_{A B}(f)\right|^{2}}{G_{A A}(f) G_{B B}(f)} .
$$

\section{MEASUREMENT SYSTEM}

A spectrum analyzer measurement system was created with the use of LabVIEW 10.0 development environment and FPGA hardware technology from National Instruments, which allows solving problems of experimental acquisition and identification of the dynamic properties of a mechanical object (fig. 4).

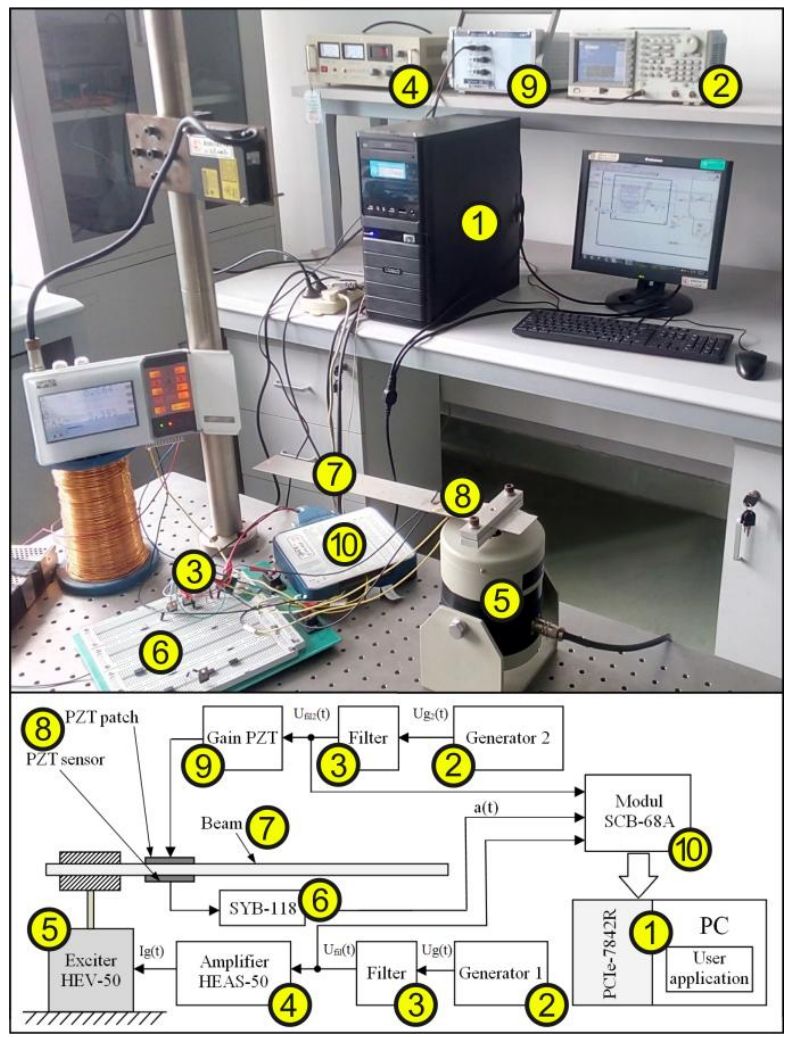

Fig. 4: General picture and scheme of the measurement system.

Automatic measurement system includes:

1. A PC equipped with a dual-channel analogue-digital transformation device based on PCIe-7842R and developed software;

2. Tektronix AFG signal generator (output $-\mathrm{Ug}(\mathrm{t}), \mathrm{V})$;

3. Analogue low pass filter (input - Ug(t), V, output Ufil (t));

4. HEAS-50 amplifier (input - Ufil(t), V; output $\operatorname{Ig}(\mathrm{t}), \mathrm{A})$;

5. HEV-50 exciter (input $-\operatorname{Ig}(\mathrm{t}), \mathrm{A}$; output $-\mathrm{F}(\mathrm{t}), \mathrm{N})$;

6. Mounting connection board;

7. The plate being studied; 
8. Pzt sensor (input - acceleration, $\mathrm{mm} / \mathrm{s}^{2}$; output $\mathrm{Ud}(\mathrm{t}), \mathrm{V})$;

9. Gain PZT XE501-A (input - Ufil2(t), V; output $\mathrm{u}(\mathrm{t}), \mathrm{V})$ );

10. SCB-68A interface module;

The software developed for the measurement system presupposes an ability to carry out harmonic analysis with the allocation of the main frequency; amplitude calculation, transfer coefficient calculation and, allotted frequency phase calculation. There is a "Signal Analysis" tab for stimulating signal spectral characteristics and response. There is a "System Analysis" tab for system frequency characteristics in FRF, Phase and Coherence.

\section{EXISTING PRINCIPLES OF VIBRATION CONTROL}

Frequency characteristics of the system on disturbance and control channels were acquired with the help of the measurement boards, the configurations of which are shown in fig. 5. Double integration was used for the output displacement signal.

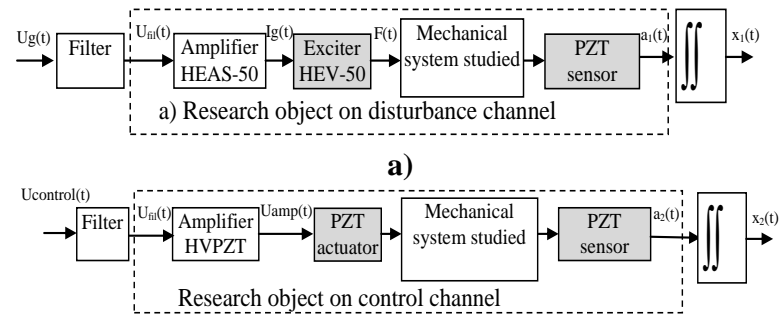

b)

Fig. 5: Structural scheme of the measurement system by channel a) - disturbance; b) - control.

\subsection{On disturbance channel}

Experimentally obtained amplitude frequency response graph (fig. 6) shows multiple pronounced resonances in [1, 2000] $\mathrm{Hz}$ frequency range. In $[2000,10000] \mathrm{Hz}$ frequency range the system is on the noise level, which is concurred by the coherence function, which reduces to zero.

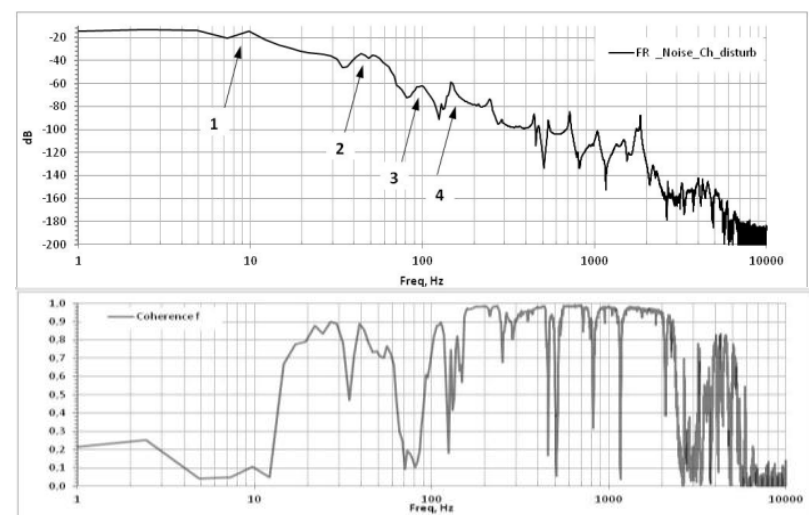

Fig. 6: Experimentally obtained frequency characteristics of the object on disturbance channel

Because of a big amount of noise in the low frequency region, frequency functions were rectified in the $[0.1,100] \mathrm{Hz}$ range during harmonic excitation. Frequency response function analysis, shows three resonances on $10,19,65 \mathrm{~Hz}$ frequencies. Those resonances will be used for the further identification of the mechanical system.

\subsection{On control channel}

On the graph of the experimentally obtained amplitude frequency response function (fig.7) on control channel in [1, 200] $\mathrm{Hz}$ frequency range there are a few resonances with a high peak value. Yet the coherence function testifies the presence of the noises, which requires additional research in this frequency domain. There are multiple pronounced resonances in the frequency range of $[200,2000] \mathrm{Hz}$. Yet their level is much lower compared to the level in the frequency response range $[1,200] \mathrm{Hz}$, which allows to not take them into consideration when identifying the model. On frequencies above $2000 \mathrm{~Hz}$ the system is on the noise level.

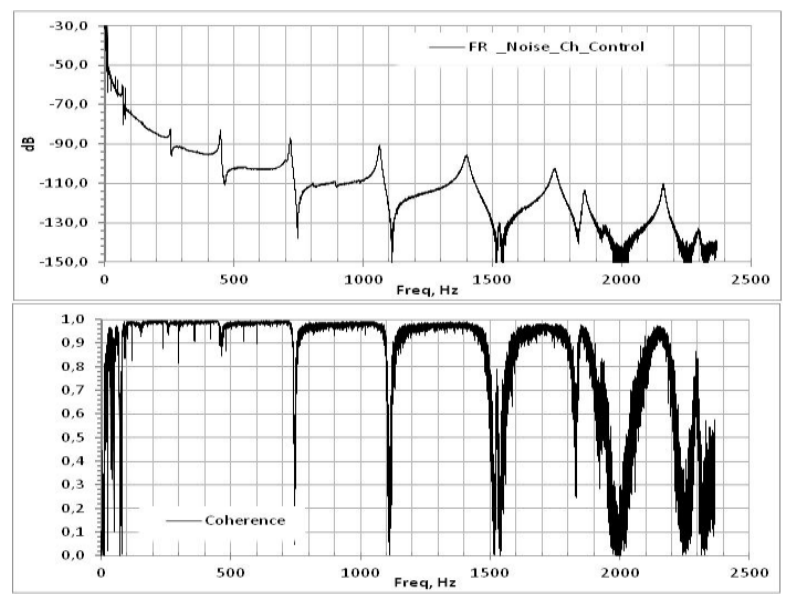

Fig. 7: Frequency characteristics on the control channel.

According to the frequency characteristics obtained, structural and parametric identification of the object was made on control and disturbance channel.

\section{IDENTIFICATION}

Experimental data was simplified towards simple calculation schemes in form of combined masses, stiffnesses, damping elements and external forces' vectors. In order to create a system of common differential equations of motion according to the calculation scheme, distributed masses were replaced with the focused ones, fig. 8 .

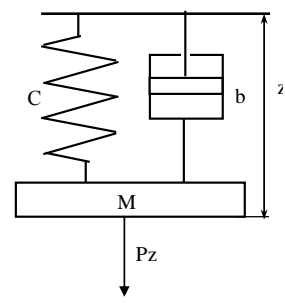

Fig. 8: Mechanical oscillation circuit

The construction of the mathematical model of oscillatory links (fig. 8) was performed with the approximation of experimentally obtained frequency characteristics of an elastic system by nonlinear programming $[9,10]$. Because of the practical inability to identify all the resonances found, their number is limited according to a certain criteria. For example, on the disturbance channel, the transmission coefficient value of the system researched on the first resonance (fig. 6) exceeds the transmission coefficient value on the fourth resonance and on all the following ones more than $40 \mathrm{~dB}$. Within displacement values it means that the oscillations on the first resonance are 20 times higher than those on the fourth one at the same excitement level. According to this, the frequency range of the system study was limited to $100 \mathrm{~Hz}$. 


\subsection{On disturbance channel}

Consideration of the frequency characteristics as of a set of vibration links (second-order differential equations) allows for the creation of a structural scheme as shown in fig. 9. The use of the multiobjective function (vector) of goal achievement from the Optimization Toolbox Matlab package allowed obtaining the estimates of the parameters of the proposed model. Bode diagram of the model is represented in fig. 10.

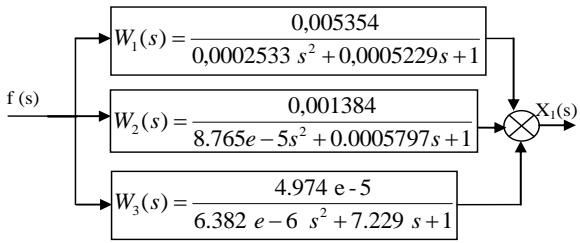

Fig. 9: Mathematical model of the system on disturbance channel

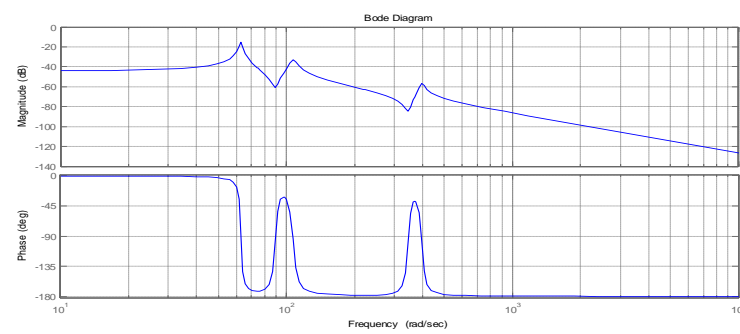

Fig. 10: Bode diagrams on disturbance channel

\subsection{On control channel}

Consideration of the frequency characteristics on the control channel allowed for the creation of a structural scheme as shown in fig. 11. Bode diagram of the model acquired is on fig. 12 .

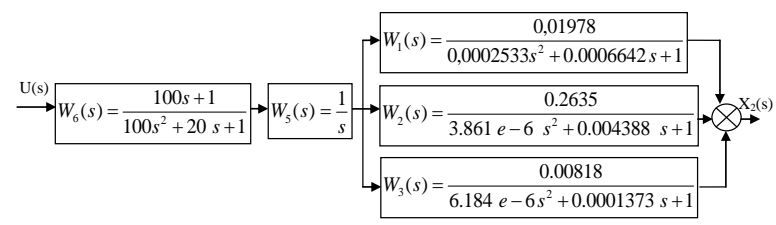

Fig. 11: Mathematical model of the system on control channel

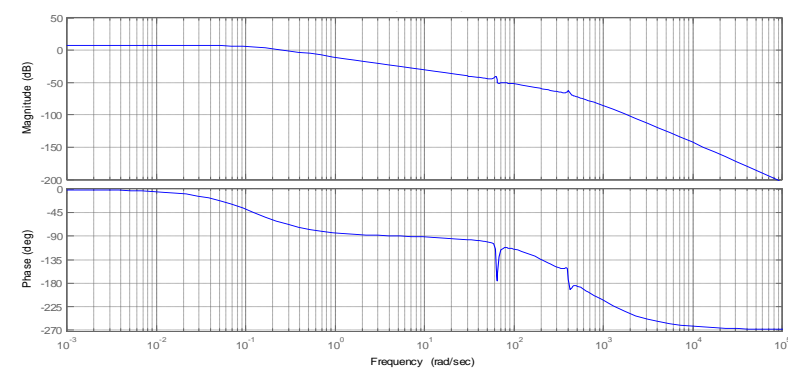

Fig. 12: Bode diagrams on control channel

\section{SYNTHESIS OF CONTROL SYSTEMS}

There were three types of feedback-based control systems with various regulators synthesized for the obtained and approximated dynamic characteristics. The effectiveness of the modeled systems' control was estimated according to the coefficient equal to the variance of the controlled variable without the control system to the variance of this variable with the control system working.

\subsection{PID regulator control system}

Finding PID coefficients for the discrete control system fig. 13 was carried out by the method of nonlinear programming, taking into account the minimization of the variance of the controlled variable from the disturbance influence of limited Effectiveness coefficients of the system at the regulator coefficients found $\left(\mathrm{k}_{\mathrm{p}}=0.5, \mathrm{k}_{\mathrm{i}}=11, \mathrm{k}_{\mathrm{d}}=0\right)$ are represented in table 1 .

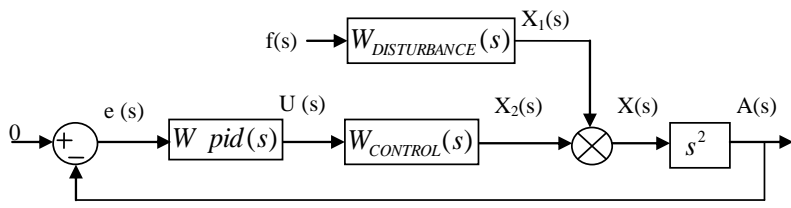

Fig. 13: Structural scheme of a PID regulator control system

The synthesized PID regulator control scheme provided transmission coefficient decrease on the disturbance channel in the frequency range of $\left[10^{-1}, 5^{*} 10^{2}\right] \mathrm{rad} / \mathrm{s}$ compared to the object without a regulator (fig. 14). The system is effective in this range; disturbance transmission coefficient decreases.

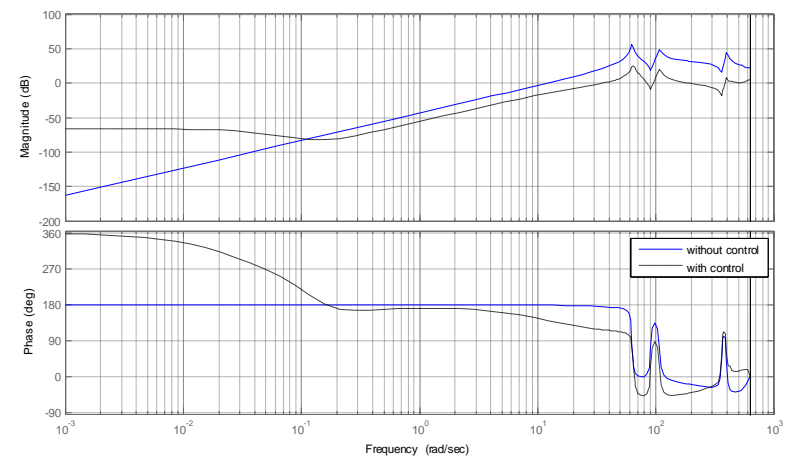

Fig. 14: Comparison of frequency characteristics of disturbance channel with and without control system

The result of the system work modeling at harmonic disturbance on the first resonance of the object is shown in fig. 15
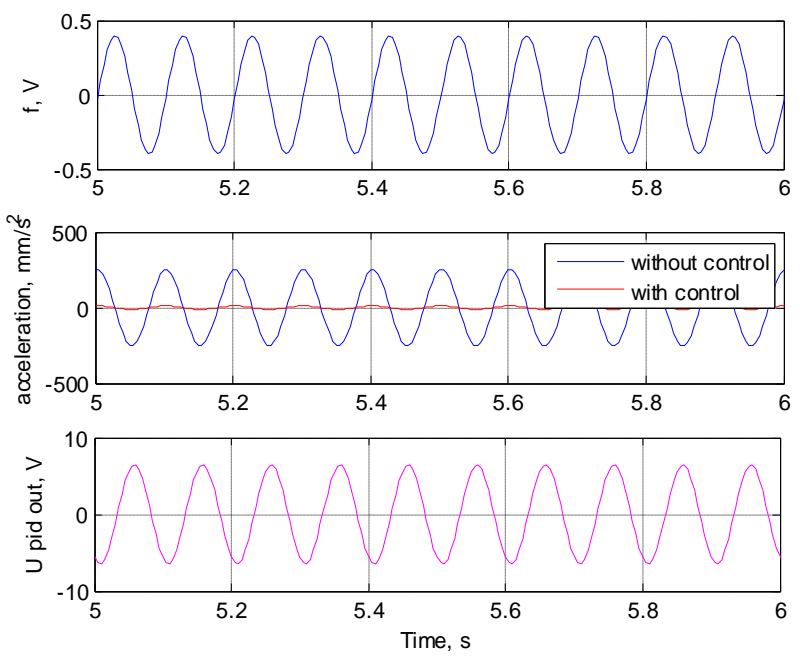

Fig. 15: The result of the modeling of the control system at the disturbance in form of the $10 \mathrm{~Hz}$ harmonic signal 


\subsection{Synthesis of a Linear Quadratic Regulator}

Synthesis of the LQG regulator (fig. 16) was done in the Control System Toolbox package of the MatLAB system [11]. The realization of the LQG regulator on the existing equipment will be carried out with ADC, DAC and an FPGAbased microcontroller, so the model of the system (fig. 16) will be discrete. The control object acquired is transferred to the state space and has the following matrix dimensions: $A=[15 \times 15], B=\left[\begin{array}{lll}15 & x & 2\end{array}\right], C=\left[\begin{array}{lll}2 & x & 15\end{array}\right], D=\left[\begin{array}{lll}2 & x & 2\end{array}\right]$. System input $f(s)$ receives input impact of a white noise type with the zero mean, from which disturbing influences with set characteristics are formed inside the model. The aim is to develop an optimal linear regulator. The average value of the quadratic functional

$$
J(u)=E \sum_{n=1}^{\infty}\left(y[n]^{T} R_{1} y[n]+u[n]^{T} R_{2} u[n]\right) .
$$

is used as the optimality criterion. Matrices of the weight coefficients $R_{1}$ and $R_{2}$, which determine the input of the original object coordinates $y[n]$ into the integral control error, and the ratio of the system error and the power of the controlling influences $u[n]$ were selected during the regulator synthesis process with a condition that the controlling signal is in the set range.

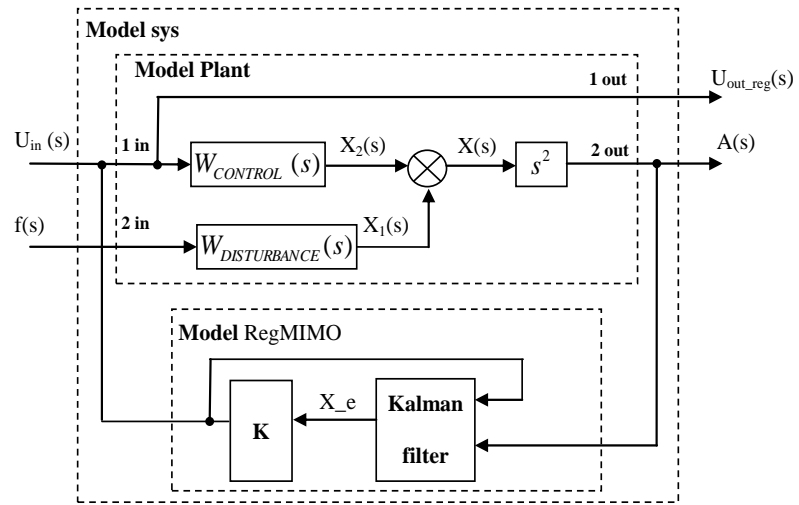

Fig. 16: Structural scheme of a system with an LQG regulator

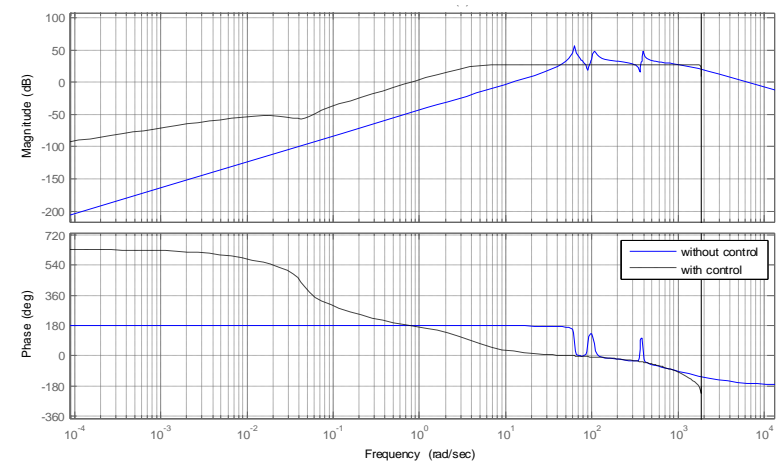

Fig. 17: Comparison of frequency characteristics of disturbance channel with and without control system

The LQG regulator control scheme provided transmission coefficient decrease on the disturbance channel in the frequency range of $\left[4 * 10^{1}, 10^{3}\right] \mathrm{rad} / \mathrm{s}$ compared to the object without a regulator (fig. 17). The system is effective in this range. Fig. 18 shows the result of the system working with the disturbing influence of the "white noise" type. Effectiveness coefficients are shown in table 1 .
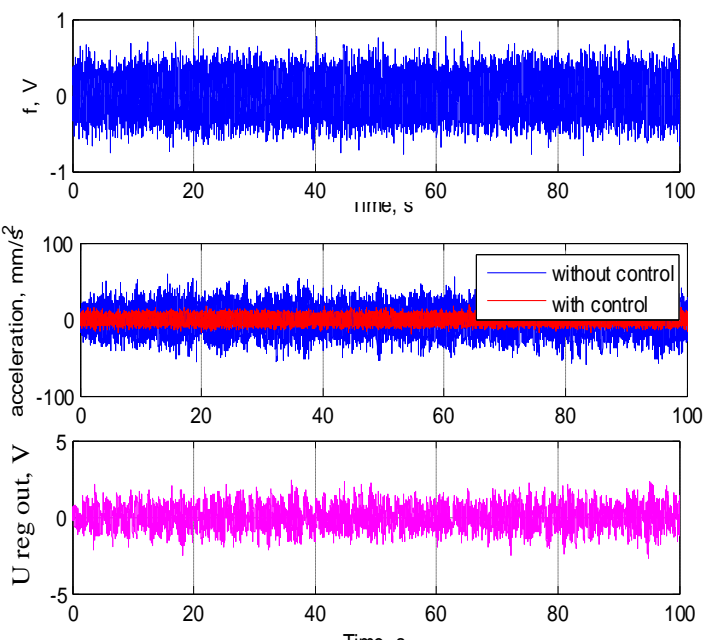

Fig. 18: The result of the modeling of the system with an LQG regulator at the disturbance from the "white noise" type signal

\subsection{Synthesis of a Linear Quadratic \\ Regulator for a Modified Object}

To research a possibility of the control system effectiveness increase with the white noise disturbance influence, the control object can be changed, and measurement and shift control, rather than acceleration can be demanded (fig. 19). When synthesizing the regulator, demand the stabilization of the "shift" parameter on the $2^{\text {nd }}$ output can be demanded. $3^{\text {rd }}$ output - acceleration A(s) is left there for control.

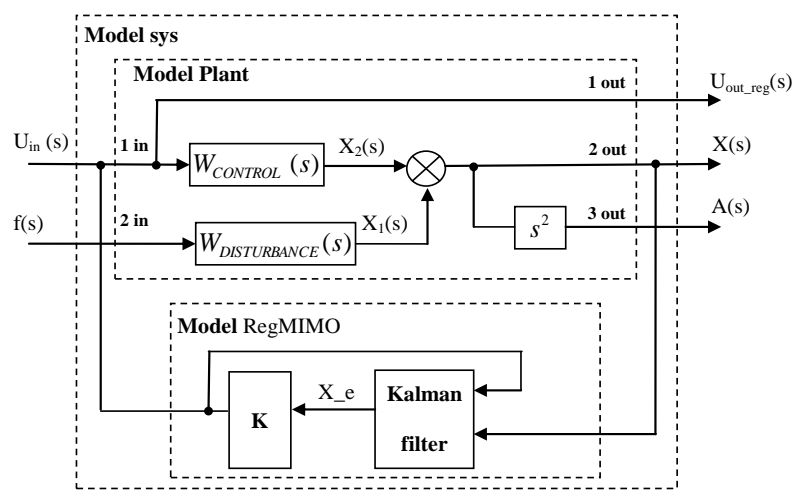

Fig. 19: Structural scheme of a system with an LQG regulator for a modified object

Amplitude-frequency characteristics on acceleration can be studied. In the frequency range of $\left[10^{-1}, 10^{3}\right] \mathrm{rad} / \mathrm{s}$ the LQG regulator control system provided a decrease of the disturbance channel signal transmission coefficient compared to the system without a regulator. The system is effective in this range. Effectiveness coefficients are shown in table 1 

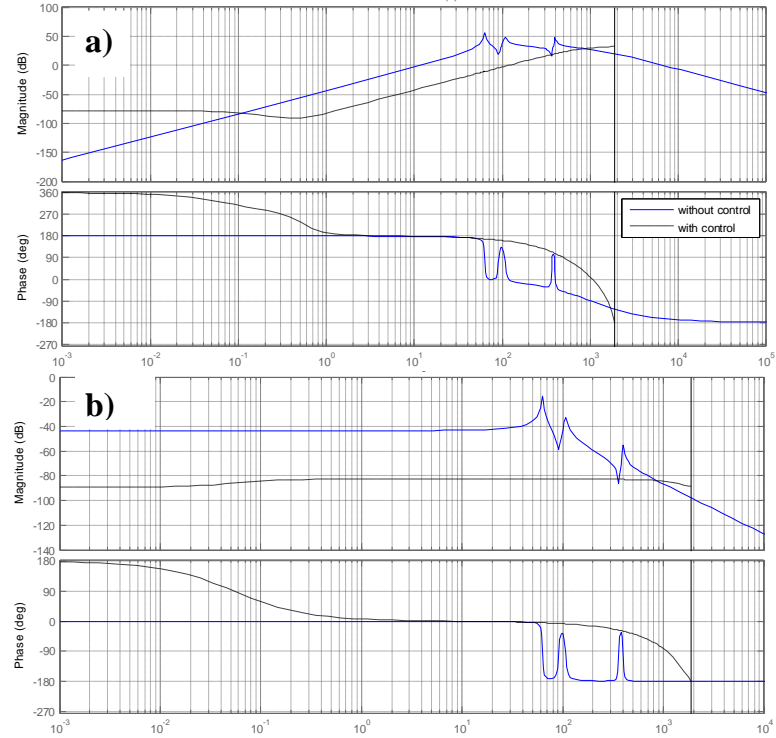

Fig. 20: Comparison of frequency characteristics of disturbance channel with and without control system (a acceleration, $b$ - displacement).

\subsection{Control system comparison}

Table 1 shows effectiveness coefficients for the synthesized systems with the disturbing influence on the first $10 \mathrm{~Hz}$ resonance of the object and with the "white noise" signal influence.

Table 1. Effectiveness coefficients for the control systems

\begin{tabular}{|c|l|c|c|}
\hline \multirow{2}{*}{ № } & \multirow{2}{*}{ Control system } & \multicolumn{2}{|c|}{ Effectiveness coefficient } \\
\cline { 3 - 4 } & $\begin{array}{l}\text { Harmonic } \\
\text { influence }\end{array}$ & $\begin{array}{c}\text { White } \\
\text { noise }\end{array}$ \\
\hline 1 & PID & 20 & 14 \\
\hline 2 & LQG type 1 & 28 & 3,5 \\
\hline 3 & LQG type 2 & $2.2 \mathrm{e}+3$ & 22 \\
\hline
\end{tabular}

Comparative analysis of the systems' work at the modeling stage in equal conditions shows that the system built around the LQG regulator, when measuring and shift controlling, is more effective than the systems based on calculation and acceleration control.

\section{CONCLUSION}

In this article:

- an electromechanic system based on the cantilever fixed plate lab equipment is studied as a control object with input and output signals differentiated;

- a vibration distribution channel - disturbance channel - was defined; and a control channel - a way for control influence - was created;

- software for the experimental definition of the dynamic properties of an electromechanic system was developed; and the dynamic properties were measured;

- structural and parametric identification of the experimental data on control and disturbance channels of the object was carried out;

- -discrete regulators with different object and regulator structures (PID, LQG) were synthesized;

- system effectiveness was compared acceleration control.

Identification of the object studied and modeling different variants of control systems for it at disturbing influence allows showing a possibility of creating an effective vibration control system. Further development of this study is realization of synthesized digital hardware regulators, their adaptation for the object and usage of the suggested approach for damping of more complex mechanical structures, as well as the search for more effective and robust control algorithms.

\section{ACKNOWLEDGMENTS}

This work was sponsored by Qing Lan Project and a Project funded by the PAPD. The authors are also grateful to the Fundamental Research Funds for the Central Universities (No. NS2013010).

\section{REFERENCES}

[1] Ehtesham Mustafa Qureshi, Xing Shen, JinJin Chen. 2014 Vibration Control laws via Shunted Piezoelectric Transducers: A Reivew. Int'1 J. of Aeronautical \& Space Sci, 15 (1).

[2] C. Richard, D. Guyomar, D. Audigier, and H. Bassaler, 2000 Enhanced semi passive damping using continuous switching of a piezoelectric device on an inductor, in Proc. SPIE Smart Struct. Mater. Conf., Passive Damping and Isolation, pp. 288-299.

[3] Donald, J.L. 2007 Engineering analysis of smart material systems John-Wiley \& Sons, Inc.: Hoboken, New Jersey.

[4] Srinivasan, A. McFarland, D.M., 2000 Smart structures: analysis and design. Cambridge University Press.

[5] Cunefare, K. A. 2000 State-switched absorber for vibration control of point-excited beams," in ASME International Mechanical Engineering Conference and Exposition, Orlando, FL.

[6] Box, D., Watts, D. 1974 Time series analysis. Prognosis and Control, Issue 1, Moscow press, pp.- 406.

[7] Box, D., Watts, D. 1974 Time series analysis. Prognosis and Control, Issue 2, Moscow press, pp.- 198.

[8] Bendat, J.S. and Piersol, A.G. 1971 Analysis and Measurement Procedures', Wiley Interscience, New York

[9] Grop, D. 1979 Methods of Identification of Systems. World, Moscow.

[10] Deutsch, A.M. 1979 Identification methods of dynamic objects, Moscow press, pp.- 240.

[11] Lazarev, Yu. 2005 Modelling of the processes and system in MatLab. The educational course. SPb: Publishing group BHV, p. 512

[12] Nikiforov, A. S., Budrin, S.V. 1968 Propagation and absorption of vibration on ships. Leningrad, «Sudostroenie», 1968 